\title{
Desenvolvimento Neuropsicomotor e Falhas Antropométricas em Crianças Menores de Dois Anos
}

\author{
Maísa de Lima Claro, ${ }^{1}$ Luisa Helena de Oliveira Lima, ${ }^{2}$ \\ Artemízia Francisca de Sousa, ${ }^{2}$ Wolney Lisboa Conde, ${ }^{2}$ \\ Luís Eduardo Soares dos Santos, ${ }^{3}$ Hengrid Graciely Nascimento Silva, ${ }^{2}$ \\ Ana Roberta Vilarouca da Silva²
}

\section{RESUMO}

Este trabalho teve por objetivo investigar a associação entre o estado nutricional e o neurodesenvolvimento psicomotor de lactentes. Consistiu em um estudo transversal, realizado com crianças na faixa etária de 1 mês a 23 meses e 31 dias, residentes no município de Picos/Piauí, sem diagnóstico prévio de atraso no neurodesenvolvimento. As variáveis investigadas foram os dados socioeconômicos, demográficos bem como os antropométricos, por meio da aferição do peso e comprimento para posterior classificação e categorização das falhas. A avaliação do neurodesenvolvimento foi realizada utilizando os Marcos de Desenvolvimento contidos na Escala de Levantamento de Bem-Estar de Crianças Pequenas. Posteriormente, realizou-se a análise estatística descritiva, além da Razão de Verossimilhança a um nível de significância de 5\%, para avaliar a associação entre a variável de desfecho e as falhas antropométricas. Das crianças avaliadas, 9,4\% apresentaram triagem positiva para a supeita de atraso no desenvolvimento neuropsicomotor, sendo a categoria 3 das falhas antropométricas a que apresentou maior prevalência $(9,1 \%)$. Neste trabalho não foi verificada associação estatística entre o estado nutricional, mediante o uso de falhas antropométricas, e os atrasos no neurodesenvolvimento infantil. Desta forma, conclui-se que, embora não tenha apresentado associação estatística, ambas as ferramentas de triagem adotadas se mostraram viáveis tanto para avaliar quanto para nortear as intervenções, visando a melhorar o estado nutricional e o neurodesenvolvimento infantil e reforçando a importância da realização deste monitoramento mais detalhado.

Palavras-chave: desenvolvimento infantil; Antropometria; estado nutricional; criança.

\section{NEUROPSYCHOMOTOR DEVELOPMENT AND ANTHROPOMETRIC FAILURES IN CHILDREN UNDER TWO YEARS}

\section{ABSTRACT}

This study aimed to investigate the association between nutritional status and infants' psychomotor neurodevelopment. It consisted of a cross-sectional study, carried out with children aged 1 month to 23 months and 31 days, living in the municipality of Picos / Piauí, without previous diagnosis of neurodevelopmental delay. The variables investigated were socioeconomic, demographic, as well as anthropometric data by measuring weight and length, for later classification and categorization of failures. The neurodevelopment assessment was performed using the Developmental Milestones contained in the Small Child Welfare Survey Scale. Subsequently, a descriptive statistical analysis was carried out, in addition to performing the Likelihood Ratio, at a significance level of $5 \%$ to assess the association between the outcome variable with anthropometric failures. Of the children evaluated, $9.4 \%$ had a positive screening for the suspected delay in neuropsychomotor development, with category 3 of the anthropometric failures having the highest prevalence (9.1\%). In this work, no statistical association was verified between nutritional status, through the use of anthropometric failures, with delays in child neurodevelopment. Thus, it is concluded that although there was no statistical association, both adopted screening tools proved viable both to assess and to guide interventions, aiming to improve nutritional status and child neurodevelopment and reinforcing the importance of carrying out this monitoring more detailed.

Keywords: child development; Anthropometry; nutritional status; child.

\footnotetext{
${ }^{1}$ Autora correspondente. Universidade Federal do Piauí (Ufpi) - Campus Universitário Ministro Petrônio Portella. Bairro Ininga. Teresina/PI, Brasil. CEP 64049-550.maisaclaro_lima@hotmail.com

2 Universidade Federal do Piauí (Ufpi). Teresina/PI, Brasil.

${ }^{3}$ Universidade Federal do Ceará (Ufce). Fortaleza/CE, Brasil.
} 


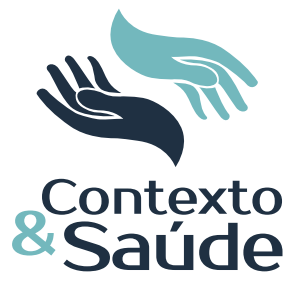

\section{INTRODUÇÃO}

Realizar a vigilância do desenvolvimento infantil nos primeiros anos de vida integra as atividades do cuidado à saúde da criança, sendo imprescindível para sua promoção, prevenção de agravos e identificação de atrasos neuropsicomotores (BRASIL, 2016). Com isso, se faz necessário acompanhar o crescimento e desenvolvimento infantil, uma vez que refletem as condições de vida tanto no momento presente quanto em longo prazo, além de contribuir na prevenção da morbimortalidade infantil (BRASIL, 2018; RODRIGUES et al., 2019).

Sendo assim, o monitoramento dos primeiros mil dias de vida da criança, que compreende o período que vai desde a concepção até os dois anos de idade, é crucial para o pleno crescimento e desenvolvimento. Nesta fase acontece o ápice da plasticidade neuronal, que depende de estímulos para que o aprendizado e a adaptação ao meio ambiente sejam estabelecidos. Desta forma, manter uma alimentação e um desenvolvimento saudável visa a preservar um estado nutricional compatível com o crescimento e com reflexos positivos durante todo o ciclo de vida do indivíduo (ALARCÓN, 2019; CUNHA; LEITE; ALMEIDA, 2015).

Além disso, o acompanhamento do crescimento infantil possibilita observar e diagnosticar precocemente desvios antropométricos, haja vista que falhas no crescimento são recorrentes, pouco estudadas e apresentam uma etiologia ampla que dificulta a triagem e o diagnóstico mais preciso (WIT; CAMP; OOSTDIJK, 2019).

Como aponta uma revisão de literatura acerca da relevância em se investigar o estado nutricional na primeira infância, tanto a má nutrição por déficit quanto por excesso tem implicações diretas na morbimortalidade infantil. Outro trabalho já evidenciou a desnutrição como sendo, dentre os fatores ambientais, a segunda causa com maior repercussão no retardo do desenvolvimento neuropsicomotor, reforçando a importância da avaliação do estado nutricional (HERNÁNDEZ et al., 2018; CARAM et al., 2006).

Desta maneira, a mensuração periódica de medidas simples, como o peso e o comprimento, possibilita um melhor acompanhamento do estado nutricional da criança, dando subsídios para se avaliar se estas medidas estão ocorrendo dentro dos padrões esperados para sua idade (FONSECA et al., 2017). Quando se identifica déficits nutricionais que comprometem o estado nutricional, este tende a interferir no desenvolvimento e na adaptação da criança ao contexto em que se encontra inserida, uma vez que, dentre os fatores ambientais, o estado nutricional tem se mostrado um dos que mais impacta o neurodesenvolvimento (HERNÁNDEZ et al., 2018).

As falhas em prosperar tornam-se perceptíveis quando a criança não atinge o padrão de crescimento esperado para o seu potencial genético em determinada faixa etária. Esses déficits podem se relacionar com atrasos nos marcos de desenvolvimento infantil. Ressalta-se que o crescimento e o amadurecimento infantil só acontecem, dentro dos padrões esperados, se a criança crescer em um ambiente saudável e estimulante com interações positivas, possibilitando, desta maneira, a aquisição oportuna das capacidades físicas, mentais e emocionais (LARSON-NATH et al., 2018; BRASIL, 2018).

Editora Unijuí - Revista Contexto \& Saúde - ISSN 2176-7114 - v. 21, n. 43, jul./set. 2021 
É importante que no monitoramento se utilizem instrumentos que permitam acompanhar e identificar precocemente qualquer alteração nos marcos do desenvolvimento infantil. Isso possibilita que as famílias, os pediatras e os demais profissionais da saúde e educação realizem intervenções eficazes em casos de atraso, como a busca por terapias mais direcionadas por meio de profissionais habilitados para este fim, de modo a reverter ou minimizar as repercussões à saúde (DIAS; PEDROSO; SANTOS, 2015; SANTANA; FILGUEIRAS; LANDEIRA-FERNANDEZ, 2015).

A Escala de Levantamento de Bem-Estar de Crianças Pequenas (do Inglês The Survey of Well-being of Young Children - SWYC) é um dos instrumentos de triagem que permite identificar tanto suspeita de atrasos no desenvolvimento neuropsicomotor quanto no comportamento, além de sondar o contexto familiar das crianças. É na primeira infância que elas estão mais propensas a apresentar e manifestar estes atrasos, sobretudo quando não recebem as devidas estimulações. Neste período apresentam uma janela de desenvolvimento mais acentuada, e por essa mesma razão também são mais susceptíveis a sofrer e receber influências ambientais, familiares, socioeconômicas e emocionais (MOREIRA, 2016; PANTOJA et al., 2018).

Os questionários do SWYC apresentam propriedades psicométricas aceitáveis para serem utilizadas tanto na vigilância do desenvolvimento da criança quanto em triagens mais rápidas com resultados satisfatórios (ARAUJO et al., 2019; MOREIRA et al., 2018).

Considerando as interlocuções entre o crescimento e o desenvolvimento infantil no início da vida, o presente estudo teve por objetivo investigar a associação entre o estado nutricional e o neurodesenvolvimento psicomotor de lactentes.

\section{MATERIAIS E MÉTODOS}

Estudo transversal realizado no período de outubro a novembro de 2019 nas Unidades Básicas de Saúde (UBS) com as Estratégias de Saúde da Família (ESF) e no Pronto-Atendimento Infantil Municipal da cidade de Picos/Piauí, que faziam acompanhamento de crianças na faixa etária do estudo de interesse (1 mês e zero dias a 23 meses e 31 dias). Após a realização do cálculo amostral de Miot (2011), com acréscimo de 10\% para fins de perdas, definiu-se a avaliação de 287 crianças. Adotou-se o erro amostral máximo de 5\% e o nível de confiança de $95 \%$.

Os critérios de elegibilidade foram: a criança encontrar-se na referida faixa etária, estar cadastrada nas ESFs do município, não apresentar ou ter sido diagnosticada com síndrome ou deficiência, estar acompanhada da mãe no momento da coleta, além dos responsáveis pela criança consentirem que ela participe da pesquisa.

Já para a avaliação do desenvolvimento neuropsicomotor, foram utilizados os Marcos do Desenvolvimento obtidos mediante a aplicação da Escala de Levantamento de Bem-Estar de Crianças Pequenas, instrumento de acesso gratuito, que pode ser aplicado da faixa etária de 1 mês a 65 meses (PERRIN et al., 2016).

Editora Unijuí - Revista Contexto \& Saúde - ISSN 2176-7114 - v. 21, n. 43, jul./set. 2021 
O mesmo possui 12 questionários destinados a diferentes faixas etárias (WHITESELL; SARCHE; TRUCKSESS, 2015). Para o presente estudo utilizou-se os questionários de 2, 4, 6, 9, 12, 15, 18 e 24 meses.

Para análise realizou-se o somatório advindo das respostas do cuidador para cada item da escala, que poderiam assumir três diferentes valores: " 0 ", quando a criança "ainda não realiza" determinada tarefa; "1", quando realiza "um pouco"; e "2", quando realiza "muito". Após a soma destas pontuações foi feita uma comparação com os escores de referência para crianças brasileiras, verificando se a criança encontrava-se acima ou abaixo do corte determinado para aquela faixa etária. Valores maiores indicavam que a criança investigada atendia às expectativas para a idade (triagem negativa), já valores iguais ou menores indicavam que a criança deveria ser encaminhada para uma avaliação diagnóstica, uma vez que se apresentava com suspeita de atraso no desenvolvimento neuropsicomotor (triagem positiva) (MOREIRA, 2016; PERRIN et al., 2016).

Vale ressaltar que apenas 213 crianças foram classificadas quanto aos Marcos do Desenvolvimento, posto que para a faixa etária de 1 a 3 meses não há pontos de corte definidos. Segundo as autoras da escala original, não foi possível determiná-los em virtude da presença de atrasos no desenvolvimento nesta referida faixa etária ser rara (SHELDRICK; PERRIN, 2013).

No estado nutricional foram coletadas as variáveis antropométricas peso e comprimento das crianças. A aferição se deu no momento da coleta, adotando os protocolos da World Health Organization (2006) para a classificação. Desta forma, os pontos de corte do escore-Z, tanto do peso quanto do comprimento, foram cruzados com a idade, além de cruzar o peso e o comprimento (BRASIL, 2011).

O peso para a idade (P/I) foi categorizado em muito baixo (< escore-z-3), baixo ( $\geq$ escore-z-3 e < escore-z-2), adequado ( $\geq$ escore-z-2 e $\leq$ escore- $z+2$ ) e elevado (> escore- $z+2$ ). O comprimento para a idade $(C / I)$ recebeu a classificação de muito baixo (< escore-z-3), baixo ( $\geq$ escore-z-3 e < escore-z-2) e adequado ( $\geq$ escore-z-2) e o peso por comprimento $(P / C)$ foi categorizado em magre$z a$ acentuada ( $<$ escore- $z-3$ ), magreza ( $\geq$ escore- $z-3$ e $<$ escore- $z-2$ ), eutrofia ( $\geq$ escore- $z-2$ e $\leq$ escore- $z+1$ ), risco de sobrepeso ( $\geq$ escore- $z+1$ e $\leq$ escore- $z+2$ ), sobrepeso ( $\geq$ escore- $z+2$ e $\leq$ escore- $z+3$ ) e obesidade ( $>$ escore- $z+3$ ) (BRASIL, 2011).

Após esta classificação, estes dados foram tabulados em planilhas do $E x$ cel for Windows ${ }^{\circledR}$ para caracterização das falhas antropométricas segundo Jeong, Kim, Subramanian (2019), que as ordenaram em sete categorias, uma vez que este modelo de avaliação pautou-se na repercussão que os déficits nutricionais podem apresentar em relação ao desenvolvimento, sendo os critérios de classificação adotados expostos na Tabela 1. 
Tabela 1 - Modelo de classificação adotado para categorização das falhas antropométricas

\begin{tabular}{|c|c|c|}
\hline Categorias & Parâmetros & Escore-z \\
\hline 1: Nenhuma falha & $\begin{array}{l}\text { P/l e C/I acima do considera- } \\
\text { do adequado }\end{array}$ & $\begin{array}{l}\mathrm{P} / \mathrm{I}>\text { escore }-\mathrm{z}+2 \\
\mathrm{C} / \mathrm{I} \geq \text { escore }-\mathrm{z}+2\end{array}$ \\
\hline $\begin{array}{l}\text { 2: C/I abaixo do } \\
\text { esperado }\end{array}$ & $\begin{array}{l}\mathrm{C} / \mathrm{I}<-2 \mathrm{SD} \text {, mas o peso era } \\
\text { aceitável tanto para a idade } \\
\text { quanto para a baixa estatura }\end{array}$ & $\begin{array}{l}C / I<\text { escore-z- } 3 ; \geq \text { escore- } z-3 \text { e }< \\
\text { escore-z-2 }\end{array}$ \\
\hline 3: Apenas magreza & $\begin{array}{l}\mathrm{P} / \mathrm{I} \text { e } \mathrm{C} / \mathrm{I} \text { adequados, mas com } \\
\mathrm{P} / \mathrm{C}<-2 \mathrm{SD}\end{array}$ & $\begin{array}{l}\mathrm{P} / \mathrm{C}<\text { escore- } \mathrm{z}-3 ; \geq \text { escore }-\mathrm{z}-3 \text { e }< \\
\text { escore-z-2 }\end{array}$ \\
\hline $\begin{array}{l}\text { 4: Apenas baixo } \\
\text { peso }\end{array}$ & $\begin{array}{l}\mathrm{P} / \mathrm{I}<-2 \mathrm{SD} \text {, mas o } \mathrm{C} / \mathrm{I} \text { e o } \mathrm{P} / \mathrm{C} \\
\text { estavam adequados }\end{array}$ & $\begin{array}{l}\mathrm{P} / \mathrm{I}<\text { escore-z- } 3 ; \geq \text { escore- } z-3 \text { e }< \\
\text { escore- } z-2\end{array}$ \\
\hline $\begin{array}{l}\text { 5: C/I e o } \mathrm{P} / \mathrm{I} \text { abai- } \\
\text { xo do recomen- } \\
\text { dado }\end{array}$ & $\begin{array}{l}\mathrm{C} / \mathrm{I} \text { e } \mathrm{P} / \mathrm{I}<-2 \mathrm{SD} \text {, mas o } \mathrm{P} / \mathrm{C} \\
\text { estava adequado }\end{array}$ & $\begin{array}{l}C / I<\text { escore- } z-3 ; \geq \text { escore- } z-3 \text { e }< \\
\text { escore-z-2 } \\
P / I<\text { escore-z- } 3 ; \geq \text { escore- } z-3 \text { e }< \\
\text { escore-z-2 }\end{array}$ \\
\hline $\begin{array}{l}\text { 6: Magreza e abai- } \\
\text { xo do peso }\end{array}$ & $\begin{array}{l}C / I \text { adequado, mas o } P / I \text { e o } \\
P / C \text { são }<-2 D\end{array}$ & $\begin{array}{l}\mathrm{P} / \mathrm{I}<\text { escore-z-3; } \geq \text { escore-z-3 e }< \\
\text { escore-z-2 } \\
\mathrm{P} / \mathrm{C}<\text { escore-z-3; } \geq \text { escore-z-3 e }< \\
\text { escore-z-2 }\end{array}$ \\
\hline $\begin{array}{l}\text { 7: Comprimento } \\
\text { baixo, magreza e } \\
\text { o peso abaixo do } \\
\text { esperado ( } 3 \text { falhas } \\
\text { simultâneas) }\end{array}$ & $\begin{array}{l}\mathrm{C} / \mathrm{I} \text { baixo e muito baixo, } \mathrm{P} / \mathrm{I} \\
\text { baixo e muito baixo e } \mathrm{P} / \mathrm{C} \text { ma- } \\
\text { greza acentuada e magreza }\end{array}$ & $\begin{array}{l}\mathrm{C} / \mathrm{I}<\text { escore-z- } 3 ; \geq \text { escore- } \mathrm{z}-3 \text { e }< \\
\text { escore-z-2 } \\
\mathrm{P} / \mathrm{I}<\text { escore-z-3; } \geq \text { escore-z-3 e }< \\
\text { escore-z-2 } \\
\mathrm{P} / \mathrm{C}<\text { escore-z-3; } \geq \text { escore-z-3 e }< \\
\text { escore-z-2 }\end{array}$ \\
\hline
\end{tabular}

Fonte: JEONG; KIM; SUBRAMANIAN (2019).

No momento da coleta foi utilizado o aplicativo EpiCollect5 ${ }^{\circledR}$, formato csv, que tem acesso gratuito, visando a tornar a pesquisa em campo mais prática. Após um contato prévio com a mãe para esclarecimentos acerca da pesquisa, caso ela consentisse participar, era solicitado a assinatura do Termo de Consentimento Livre e Esclarecido (TCLE) de acordo com a idade materna.

Para as análises estatísticas os dados foram transferidos ao Software for Statistical Package for the Social Sciences (IBM SPSS Statistics Base 20.0). Utilizou-se a estatística descritiva para obtenção das frequências absolutas e relativas. Para avaliar a associação entre a variável de desfecho (atraso no neurodesenvolvimento infantil) com as falhas antropométricas, utilizou-se a Razão de Verossimilhança a um nível de significância de $5 \%$.

O estudo foi aprovado pelo Comitê de Ética da Universidade Federal do Piauí, sob número de parecer favorável 3.645.183, quando se levou em consideração os aspectos éticos e metodológicos de acordo com as diretrizes estabelecidas na Resolução 466/2012 e complementares do Conselho Nacional de Saúde (BRASIL, 2013).

\section{RESULTADOS}

Ao analisar os dados percebeu-se que dentre as crianças avaliadas houve predominância do sexo feminino $(50,9 \%)$ e que a faixa etária de um a três meses de idade foi a mais recorrente $(25,8 \%)$, enquanto a participação de crianças 
entre 23 a 24 meses foi a que apresentou menor expressividade (2,4\%). Em relação à frequência de atrasos no neurodesenvolvimento mediante a avaliação dos Marcos do Desenvolvimento contidos na escala SWYC, das 287 crianças avaliadas 9,4\% apresentaram triagem positiva para atraso no desenvolvimento neuropsicomotor.

Já no que se refere às falhas antropométricas, que está representada como Modelo 1, é perceptível visualizar que uma parcela significativa do público infantil avaliado não apresentou nenhuma falha $(87,5 \%)$. Destas crianças, no entanto, 9,1\% estavam com o peso abaixo do que é esperado para o comprimento dentre as categorias investigadas para caracterização do estado nutricional, mediante o uso do conceito de falhas. Todas estas informações encontram-se descritas na Tabela 2.

Tabela 2 - Perfil social, antropométrico e do neurodesenvolvimento das crianças avaliadas, Picos, Piauí $(n=287)$

\begin{tabular}{cccc}
\hline Variáveis & & $\mathbf{N}$ & \% \\
\hline \multirow{2}{*}{ Sexo } & Feminino & 146 & 50,9 \\
& Masculino & 141 & 49,1 \\
& $1-3$ & 74 & 25,8 \\
& $4-5$ & 34 & 11,8 \\
Idade das crianças (meses) & $6-8$ & 55 & 19,2 \\
& $9-11$ & 32 & 11,2 \\
& $12-14$ & 33 & 11,5 \\
& $15-17$ & 25 & 8,7 \\
Marcos do Desenvolvimento & 27 & 9,4 \\
& $18-22$ & 7 & 2,4 \\
& $23-24$ & 74 & 25,8 \\
& Não se Aplica & 27 & 9,4 \\
& Triagem Positiva & 186 & 64,8 \\
& Triagem Negativa & 251 & 87,5 \\
& Categoria 1 & 1 & 0,3 \\
& Categoria 2 & 26 & 9,1 \\
Modelo 1 ${ }^{€}$ & Categoria 3 & 2 & 0,7 \\
& Categoria 4 & 0 & 0 \\
& Categoria 5 & 6 & 2,1 \\
& Categoria 6 & 1 & 0,3 \\
\hline
\end{tabular}

€Modelo 1 = Falhas Antropométricas.

Fonte: Os autores.

Quando foi realizado o cruzamento das falhas antropométricas com os dados referentes as 213 crianças que se enquadravam na classificação dos Marcos do Desenvolvimento, foi perceptível que apenas duas crianças tiveram tanto triagem positiva para atrasos no desenvolvimento quanto para falhas antropométricas, sendo estas nas categorias magreza apenas e magras e abaixo do peso, conforme demonstrado na Tabela 3. 
Tabela 3 - Suspeitas de atraso no desenvolvimento neuropsicomotor segundo o estado nutricional, avaliado pelas falhas antropométricas $(n=213)$

\begin{tabular}{cccc}
\hline Variáveis & $\begin{array}{c}\text { Triagem Positiva } \\
\mathbf{n}(\%)\end{array}$ & $\begin{array}{c}\text { Triagem Negativa } \\
\mathbf{n}(\%)\end{array}$ & \multirow{2}{*}{ Valor $\mathbf{p}^{*}$} \\
\hline Nenhuma Falha & $25(12,7)$ & $172(87,3)$ & \\
Apenas Atrofiado & $0(0)$ & $1(100)$ & \\
Magreza Apenas & $1(7,7)$ & $12(92,3)$ & 0,287 \\
$\begin{array}{c}\text { Magras e Abaixo do Peso } \\
\text { Atrofiado, Magro e Abai- }\end{array}$ & $1(100)$ & $0(0)$ & \\
$\quad$ xo do Peso & $0(0)$ & $1(100)$ & \\
\hline
\end{tabular}

*Razão de Verossimilhança.

Fonte: Os autores.

Desta forma, na presente amostra o estado nutricional avaliado mediante as falhas antropométricas não apresentou associação estatística com atrasos no neurodesenvolvimento infantil mediante os Marcos do Desenvolvimento da escala SWYC $(p=0,287)$. O estudo demonstrou, no entanto, a importância do monitoramento destas variáveis, tendo em vista que ainda há crianças que não se encontravam dentro dos padrões de crescimento e desenvolvimento esperados para a idade.

\section{DISCUSSÃO}

Um comparativo entre os resultados encontrados para as falhas antropométricas com o estudo utilizado como base, requer um pouco de cautela na interpretação, uma vez que tanto o número amostral quanto a faixa etária das crianças são diferentes, o que pode explicar as variações encontradas. Dentre as categorias que tiveram uma maior prevalência de falhas no estudo base, o comprimento abaixo do esperado para idade apresentou $31,4 \%$, enquanto, no presente estudo, a maior prevalência foi na categoria do peso para o comprimento $(9,1 \%)$, posto que estes foram classificados com magreza, e no estudo comparativo esta mesma falha ainda apresentou um percentual maior $(16,1 \%)$ (JEONG; KIN; SUBRAMANIAN, 2019).

Pode-se notar que tanto no estudo base quanto no presente trabalho as falhas sempre manifestaram correlação com atrasos no crescimento, por isso ponderar os resultados encontrados para alterações nos déficits estaturais se faz necessário. Embora o presente trabalho não tenha apresentando uma alta incidência de atraso nesta categoria, cabe reforçar que na prevalência de crianças com falhas nas demais categorias, normalmente a estatura vinha associada. Por isto, a importância de um monitoramento mais cauteloso da estatura, uma vez que ela reflete o crescimento linear da criança, sendo um indicativo de que possa ter vivenciado ou estar vivenciando situações desfavoráveis, pois o seu efeito é acumulativo (MAGALHÃES et al., 2016).

De maneira geral, ao se analisar as falhas antropométricas vários fatores devem ser investigados numa tentativa de revertê-las, como os aspectos obstétricos, socioeconômicos e alimentares. A literatura reporta uma associação com

Editora Unijuí - Revista Contexto \& Saúde - ISSN 2176-7114 - v. 21, n. 43, jul./set. 2021 
a história pregressa da criança, em que o baixo peso ao nascer pode repercutir no estado nutricional durante a infância, sendo este uma das variáveis que mais impactam no crescimento, na composição corporal e no desenvolvimento infantil, reflexos advindos ainda do período de vida intrauterino, que traz consigo comorbidades que repercutirão na saúde na fase adulta. Além disso, os fatores intrínsecos ao ambiente em que esta criança está inserida também têm interferência (ROCHA et al., 2019; LARSSON et al., 2019; MAGALHÃES et al., 2016; SHOEPS et al., 2011; WILCOX, 2001).

Já quando se analisou o total de crianças que apresentava desvio tanto na estatura quanto no peso nas três categorias antropométricas analisadas, o presente estudo não evidenciou nenhuma criança com estas três falhas, contrariando o trabalho de Jeong, Kin, Subramanian (2019), em que 12\% de suas crianças tiveram esta sobreposição de déficits. Os autores ressaltam a importância em ponderar todos estes indicadores no momento de classificar e associar os achados ao estado nutricional, principalmente no que se refere às categorias de desnutrição infantil.

Esta ressalva deve-se ao fato de que os dois primeiros anos de vida da criança são cruciais para seu pleno crescimento e desenvolvimento, principalmente em crianças com histórico de baixo peso ao nascer e cujas famílias apresentam baixo poder aquisitivo (MAGALHÃES et al., 2016).

Embora no presente estudo as falhas antropométricas usadas para mensurar o estado nutricional não tenham apresentado associação com atrasos nos Marcos do Desenvolvimento, cabe mencionar a importância em acompanhar o crescimento e o desenvolvimento destas crianças nos dois primeiros anos de vida por meio das variáveis elencadas neste trabalho. Nesta fase o estado nutricional fica mais susceptível a oscilações, principalmente quando há alterações no ambiente em que a criança vive, podendo ocasionar uma maior vulnerabilidade que tende a repercutir na sua saúde. Por isto, o olhar para com este público deve ser biopsicossocial, estendendo o diálogo para o ambiente familiar e o contexto social onde ela está inserida (PEDRAZA; OLINDA; BEZERRA, 2020; BRASIL, 2012).

Desta forma, percebe-se que o cuidado e o acompanhamento da criança são dinâmicos e acontecem diariamente, além de envolverem a participação de vários atores sociais; dentre eles, um dos mais importantes é o que possui vínculos familiares, uma vez que os primeiros estímulos advêm deste ambiente, e são exemplos a serem seguidos pelas crianças, por isto estimulá-las é de suma relevância, haja vista que nos primeiros meses de vida dá-se início ao processo do desenvolvimento motor, da linguagem e do campo mental, e estes devem acontecer de maneira plena (OLIVEIRA; ROCHA, 2019).

É importante monitorar, também, o desenvolvimento infantil, pois, como investigado no estudo, 27 crianças ainda tiveram triagem positiva para risco de atraso no neurodesenvolvimento infantil. Quando consultada a literatura, estes resultados divergiram em termos percentuais com os encontrados por Moreira (2016), que utilizou a mesma escala de triagem e encontrou índices de risco de atraso bem mais expressivos, embora com uma amostra bem mais extensa.

Editora Unijuí - Revista Contexto \& Saúde - ISSN 2176-7114 - v. 21, n. 43, jul./set. 2021 
Ainda, é interessante salientar que, mesmo que a amostra trabalhada não tenha apresentando associação entre atrasos no desenvolvimento com as falhas antropométricas, é consenso na literatura que os hábitos alimentares têm influência na antropometria e apresentam relação com atrasos no desenvolvimento, como reporta uma pesquisa norte-americano, que avaliou crianças na faixa etária de até 4 anos de idade e percebeu que quanto mais velhas maiores são os riscos de insegurança alimentar, e este tende a refletir em atrasos no desenvolvimento, considerando que nele não foi constatado associação em alterações antropométricas (DRENNEN et al., 2019).

Estes impactos podem ser explicados partindo do princípio que durante o período gestacional a dieta da mãe tende a suprir as demandas de proteínas e nutrientes para que a mielinização aconteça de forma otimizada, à medida que quando a criança nasce esta fica na dependência de terceiros e, caso o aporte de vitamina B12 seja deficiente, o mesmo pode interferir em atrasos nas funções cognitivas (ALARCÓN, 2019).

Desta forma, é perceptível a necessidade cada vez mais crescente em investigar e acompanhar o crescimento infantil, tendo em vista que o desenvolvimento e o estado nutricional vivenciados nesta fase têm repercussões cruciais ao longo de toda sua existência, além de implicar nas futuras gerações, por isto a urgência em proporcionar condições para que estas crianças alcancem seu máximo potencial, e este deve sempre vir pautados com amor e cuidado (RICHTER et al., 2020).

\section{CONCLUSÃO}

Sendo assim, conclui-se, ao final do estudo, que, embora não tenham sido evidenciadas associações entre as falhas antropométricas e os Marcos do Desenvolvimento, ambos mostraram-se ferramentas alternativas para avaliar tanto o estado nutricional, mediante o cruzamento de distintas variáveis, que mostram resultados coerentes para o correto manejo das crianças que, porventura vierem a apresentar alguma falha, quanto para a triagem de possíveis atrasos no desenvolvimento infantil, reforçando a relevância de um acompanhamento mais detalhado.

Além disso, os resultados aqui elencados levam a refletir acerca da importância do monitoramento e replicação destes estudos em outras regiões para que seja possível uma generalização das informações, haja vista que, no modelo de estudo transversal, apenas uma amostra isolada não possibilita a extrapolação destes dados com segurança, bem como investigar, em estudos próximos, os hábitos alimentares destas crianças para verificar uma possível relação entre estas variáveis.

\section{REFERÊNCIAS}

ALARCÓN, O. T. Neurodesarrollo en los primeros 1.000 días de vida. Rol de los pediatras. Rev. Chil. Pediatr., Santiago, v. 90, n. 1, p. 11-16, 2019. Disponível em: https://scielo.coni-

Editora Unijuí - Revista Contexto \& Saúde - ISSN 2176-7114 - v. 21, n. 43, jul./set. 2021 
cyt.cl/scielo.php?script=sci_arttext\&pid=S0370-41062019000100011\&lng=es\&nrm=iso. Acesso em: 31 mar 2020.

ARAUJO, L. B. et al. Avaliação neuropsicomotora de crianças de 0 a 5 anos em centros de educação infantil do ensino público. Rev. Cefac., v. 21, n. 3, p. 1-9, 2019. Disponível em: http://www.scielo.br/pdf/rcefac/v21n3/pt_1982-0216-rcefac-21-03-e12918.pdf. Acesso em: 26 nov. 2019.

BRASIL. Ministério da Saúde. Secretaria de Atenção à Saúde. Departamento de Ações Programáticas Estratégicas. Política Nacional de Atenção Integral à Saúde da Criança: orientações para implementação. Brasília: Ministério da Saúde, 2018. Disponível em: http://www.saude.pr.gov.br/arquivos/File/Politica_Nacional_de_Atencao_Integral_a_ Saude_da_Crianca_PNAISC.pdf. Acesso em: 5 maio 2020.

BRASIL. Ministério da Saúde. Secretaria de Atenção à Saúde. Diretrizes de estimulação precoce: crianças de zero a 3 anos com atraso no desenvolvimento neuropsicomotor. Brasília: Ministério da Saúde, 2016.

BRASIL. Resolução N 466, de 12 de dezembro de 2012. Diário Oficial da União, Presidência da República, Brasília, DF, 13 de junho de 2013. Seção 1, p. 59. Disponível em: http:// bvsms.saude.gov.br/bvs/saudelegis/cns/2013/res0466_12_12_2012.html. Acesso em: 8 jun. 2017.

BRASIL. Ministério da Saúde. Secretaria de Atenção à Saúde. Departamento de Atenção Básica. Saúde da criança: crescimento e desenvolvimento. Brasília: Ministério da Saúde, 2012.

BRASIL. Ministério da Saúde. Secretaria de Atenção à Saúde. Departamento de Atenção Básica. Orientações para a coleta e análise de dados antropométricos em serviços de saúde: Norma Técnica do Sistema de Vigilância Alimentar e Nutricional - Sisvan. Brasília: Ministério da Saúde, 2011.

CARAM, L. H. A. et al. Investigação das causas de atraso no neurodesenvolvimento: recursos e desafios. Arq. Neuropsiquiatr, v. 64, n. 2, p. 466-472, 2006. Disponível em: https:// www.scielo.br/pdf/anp/v64n2b/a22v642b.pdf. Acesso em: 9 jul. 2020.

CUNHA, A. J. L. A.; LEITE, A. J. M.; ALMEIDA, I. S. The pediatrician's role in the first thousand days of the child: the pursuit of healthy nutrition and development. J. Pediatr. Rio de Janeiro, v. 91, n. 6, p. 44-51, 2015. Disponível em: http://www.scielo.br/scielo.php?script=sci_arttext\&pid=S0021-75572015000800006. Acesso em: 15 abr. 2018.

DIAS, G. B.; PEDROSO, J. S.; SANTOS, A. C. S. L. Avaliação de desenvolvimento de bebês em acolhimento institucional com "Ages and Stages Questionnaires". Mudanças - Psicologia da Saúde, v. 23, n. 2, p. 1-7, 2015. Disponível em: https://www.metodista.br/revistas/revistas-ims/index.php/MUD/article/view/6655/5274. Acesso em: 28 mar. 2018.

DRENNEN, C. R. et al. Food Insecurity, Health, and Development in Children Under Age Four Years. Pediatrics, v. 144, n. 4, 2019. Disponível em: https://pediatrics.aappublications.org/content/144/4/e20190824. Acesso em: 31 mar. 2020.

FONSECA, P. C. A. et al. Determinantes da velocidade média de crescimento de crianças até seis meses de vida: um estudo de coorte. Ciência \& Saúde Coletiva, v. 22, n. 8, p. 2.713-2.726, 2017. Disponível em: https://www.scielo.br/pdf/csc/v22n8/1413-8123csc-22-08-2713.pdf. Acesso em: 9 jul. 2020.

HERNÁNDEZ, J. A. L. et al. Estado nutricional y neurodesarrollo en la primera infancia. Rev. Cubana Salud Pública, v. 44, n. 4, p. 169-185, 2018. Disponível em: http://scielo.sld. cu/pdf/rcsp/v44n4/1561-3127-rcsp-44-04-169.pdf. Acesso em: 9 jul. 2020.

JEONG, J.; KIM, R.; SUBRAMANIAN, S. V. Multiple anthropometric failures and early child development in 34 low- and middle-income countries. J Glob Health Sci., v. 1, n. 2, p. 1-11, 2019. Disponível em: https://www.researchgate.net/publication/337596075_Multiple_anthropometric_failures_and_early_child_development_in_34_low-_and_middle-income_countries. Acesso em: 7 fev. 2020.

LARSSON, A. et al. Body composition and growth in full-term small for gestational age and large for gestational age Swedish infants assessed with air displacement plethysmography at birth and at 3-4 months of age. PLOS ONE, v. 14, n. 5, p. 1-11, 2019. Disponível em: https://www.ncbi.nlm.nih.gov/pubmed/31091240. Acesso em: 26 abr. 2020.

Editora Unijuí - Revista Contexto \& Saúde - ISSN 2176-7114 - v. 21, n. 43, jul./set. 2021 
LARSON-NATH, C. et al. Defining Pediatric Failure to Thrive in the Developed World: Validation of a Semi-Objective Diagnosis Tool. Clin. Pediatr. (Phila), v. 58, n. 4, p. 446-452, 2019. Disponível em: https://www.ncbi.nlm.nih.gov/pubmed/30596256. Acesso em: 5 maio 2020.

MAGALHÃES, E. I. S. et al. Déficit estatural e fatores associados em crianças de 6 a 24 meses atendidas em unidades de saúde do sudoeste da Bahia. Cad. Saúde Colet., v. 24, n. 1, p. 84-91, 2016. Disponível em: https://doi.org/10.1590/1414-462X201600010037. Acesso em: 25 jun. 2020.

MIOT, H. A. Tamanho da amostra em estudos clínicos e experimentais. J. Vasc. Bras., v. 10, n. 4, p. 275-278, 2011. Disponível em: http://www.scielo.br/pdf/jvb/v10n4/v10n4a01. Acesso em: 18 mar. 2019.

MOREIRA, R. S. et al. "Survey of Wellbeing of Young Children (SWYC)": how does it fit for screening development aldelay in Brazilian children aged 4 to 58 months? Research in Developmental Disabilities, v. 78, n. 1, p. 78-88, 2018. Disponível em: https://www.ncbi. nIm.nih.gov/pubmed/29793101. Acesso em: 26 out. 2019.

MOREIRA, R. S. Triagem de atraso de desenvolvimento e de alterações de comportamento: estudo normativo do "Survey of Wellbeing of Young Children (SWYC)" no contexto brasileiro. 2016. 174 f. Tese (Doutorado em Ciências da Saúde) - Faculdade de Medicina, Universidade Federal de Minas Gerais, Belo Horizonte, 2016. Disponível em: https:// repositorio.ufmg.br/bitstream/1843/BUOS-AM8PW5/1/tese_rafaela_final_corrigida.pdf. Acesso em: 20 mar. 2018.

OLIVEIRA E. A. R.; ROCHA, S. S. O cuidado cultural dos pais na promoção do desenvolvimento infantil. Rev. Fund. Care On-line, v. 11, n. esp, p. 397-403, 2019. Disponível em: http://ciberindex.com/index.php/ps/article/view/P112021. Acesso em: 25 abr. 2020.

PANTOJA, A. P. P. et al. Análise do efeito dos fatores ambientais sobre o desenvolvimento neuropsicomotor de crianças em comunidade amazônica. Journal of Human Growth and Development, v. 28, n. 3, p. 232-239, 2018. Disponível em: http://pepsic.bvsalud.org/ pdf/rbcdh/v28n3/pt_03.pdf. Acesso em: 14 nov. 2019.

PEDRAZA, D. F.; OLINDA, R. A.; BEZERRA, T. A. Estado nutricional de crianças residentes em área de vulnerabilidade social: estudo longitudinal. Saúde Debate, v. 44, n. 124, p. 130-140, 2020. Disponível em: https://doi.org/10.1590/0103-1104202012409. Acesso em: 25 jun. 2020.

PERRIN, E. C. et al. The Survey of Well-being of Young Children (SWYC) User's Manual. Version 1.01 ed. Boston: Center; Tufts Medical, 2016.

RICHTER, L. M. et al. Guidance for the health sector to partner with parents and families for early childhood development. Lancet, v. 7, v. 395, p. 766-768, 2020. Disponível em: https://www.ncbi.nlm.nih.gov/pubmed/32145775. Acesso em: 15 abr. 2020.

ROCHA, E. M. B. et al. A relação do peso ao nascer com os indicadores antropométricos em crianças em idade pré-escolar. O Mundo da Saúde, v. 43, n. 3, p. 701- 712, 2019. Disponível em: https://pesquisa.bvsalud.org/portal/resource/pt/biblio-1054516. Acesso em: 26 abr. 2020.

RODRIGUES, D. A. et al. Avaliação da adesão às consultas de crescimento e desenvolvimento infantil. Rev. Enferm. Ufpe on-line, v. 13, n. 4, p. 1.023-1.029, 2019. Disponível em: https://periodicos.ufpe.br/revistas/revistaenfermagem/article/view/238262/31801. Acesso em: 5 maio 2020.

SANTANA, C. M. T.; FILGUEIRAS, A.; LANDEIRA-FERNANDEZ, J. Ages \& Stages Questionnaire-Brazil-2011: Adjustments on an Early Childhood Development Screening Measure. Global Pediatric Health, v. 2, p. 1-12, 2015. Disponível em: https://www.ncbi.nlm.nih. gov/pmc/articles/PMC4784636/pdf/10.1177_2333794X15610038.pdf. Acesso em: 22 mar. 2018.

SHELDRICK, R. C.; PERRIN, E. C. Evidence-Based Milestones for Surveillance of Cognitive, Language, and Motor Development. Academic Pediatrics, v. 13, n. 6, p. 577-586, 2013. Disponível em: https://www.ncbi.nlm.nih.gov/pubmed/24238685. Acesso em: 18 mar. 2018.

Editora Unijuí - Revista Contexto \& Saúde - ISSN 2176-7114 - v. 21, n. 43, jul./set. 2021 
SHOEPS, D. O. et al. Nutritional status of pre-school children from low incomes families. Nutritional Journal, v. 10, n. 43, p. 1-6, 2011. Disponível em: https://www.ncbi.nlm.nih. gov/pmc/articles/PMC3112402/. Acesso em: 26 abr. 2020.

WILCOX, A. J. On the importance - and the unimportance - of birthweight. Int J Epidemiol, v. 30, p. 1.233-1.241, 2001. Disponível em: https://www.ncbi.nlm.nih.gov/pubmed/11821313. Acesso em: 26 abr. 2020.

WIT, J. M.; KAMP, G. A.; OOSTDIJK, W. Towards a Rational and Efficient Diagnostic Approach in Children Referred for Growth Failure to the General Paediatrician. Horm Res Paediatr, v. 91, n. 4, p. 223-240, 2019. Disponível em: https://www.ncbi.nlm.nih.gov/ pubmed/31195397. Acesso em: 5 maio 2020.

WHITESELL, N. R.; SARCHE, M; TRUCKSESS, C. The Survey of Well-Beingof Young Children: Results of a Feasibility Study With American indian and Alaska Native Communities. Infant Mental Health Journal, v. 36, n. 5, p. 483-505, 2015. Disponível em: https://www. ncbi.nlm.nih.gov/pubmed/26312600. Acesso em: 12 mar. 2018.

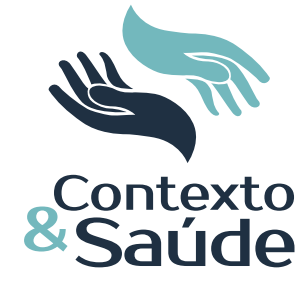

